\title{
Sozialpsychiatrie: Gegensatz zu biologischer Psychiatrie und Psychotherapie?
}

\author{
Social Psychiatry - Contradiction to Biological Psychiatry and Psychotherapy?
}

Autoren

Institute
Johannes Wancata' ${ }^{1}$, Ullrich Meise ${ }^{2}$

Universitätsklinik für Psychiatrie und Psychotherapie, Wien / Österreich

Universitätsklinik für Allgemeine Psychiatrie und Sozialpsychiatrie, Medizinische Universität Innsbruck/Österreich

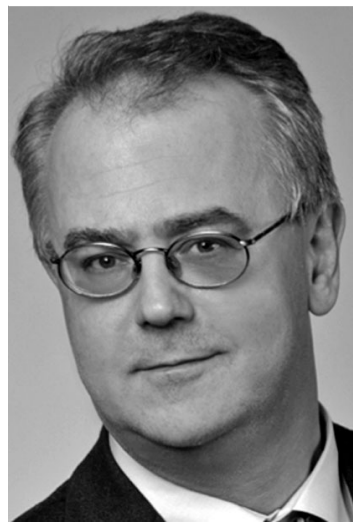

Univ.-Prof. Dr. Johannes Wancata

\section{Bibliografie}

Dol http://dx.doi.org/ 10.1055/s-0030-1248601

Psychiat Prax 2010; 37:

317-318

(c) Georg Thieme Verlag KG

Stuttgart · New York .

ISSN 0303-4259

\section{Korrespondenzadresse \\ Univ.-Prof. Dr. Johannes Wancata}

Universitätsklinik für Psychiatrie und Psychotherapie Währinger Gürtel 18-20 1090 Wien, Österreich johannes.wancata@ meduniwien.ac.at
Vor einiger Zeit wurde an dieser Stelle [1] eine neue Chance für die Entwicklung und Umsetzung psychosozialer Interventionen postuliert. Die Tatsache, dass manche psychopharmakologische Entwicklungen von einigen zu optimistisch gesehen und in bestimmten Aspekten überschätzt wurden, war der Ausgangspunkt dieser Überlegungen. Obwohl wir klare Belege für die Wirksamkeit soziotherapeutischer und psychotherapeutischer Interventionen haben [2], werden diese häufig nicht oder in zu geringem Umfang angeboten. Neben der Implementierung von Maßnahmen, deren Wirksamkeit nachgewiesen ist, muss sich aber eine wissenschaftliche Zeitschrift auch die Frage nach künftigen Forschungsfragen stellen.

Wir wissen heute, dass biologische, lebensgeschichtliche und soziale Faktoren Noxencharakter haben und auf diese Weise zur Entstehung von Krankheiten beitragen können. Der Einfluss von Umgebungsfaktoren und individuell-biografischer Erfahrung auf das neuronale Substrat ist mittlerweile nachgewiesen [3]. All unser Erleben basiert auf komplexen zentralnervösen Prozessen. So führen biografische und soziale Faktoren (z.B. traumatische Erlebnisse, soziale Isolation) zu überdauernden biologischen Veränderungen, welche wieder die Empfindlichkeit für Umweltfaktoren erhöhen oder erniedrigen. Obwohl also klar ist, dass Umgebungsfaktoren (z.B. soziales Netzwerk, das emotionale Klima, städtische Umgebung) das Auftreten, den Verlauf bzw. die Prognose von psychischen Krankheiten beeinflussen können [4], sind viele Details dieser Interaktionen noch unklar [5]. Wichtige Beispiele in diesem Kontext sind die Umwelt-Gen-Interaktionen, die vermutlich eine wichtige Grundlage neurobiologischer Anpassungsvorgänge darstellen [6].

In der Forschung sind oft umschriebene und eng definierte Forschungsansätze notwendig, wenn unter Kontrolle anderer potenzieller Einflüsse Hypothesen geprüft werden sollen. Der Vorteil der experimentellen „Überschaubarkeit“ darf aber in der wissenschaftlichen Theorienbildung nicht zu einem eingeengten Bild psychischer Erkrankungen führen, die soziale, lebensgeschichtliche und biologische Aspekte gegeneinander ausspielen. So wie es in der Psychotherapieforschung heute nicht mehr als „unanständig“ gilt, biologische und psychologische Forschungstechniken (z.B. Bildgebung und psychologische Testverfahren) zu kombinieren, könnte eine ähnliche Vorgangsweise längerfristig auch für die Sozialpsychiatrie fruchtbare Kooperationen ermöglichen. Manche Autoren fordern schon seit längerer Zeit eine stärkere Integration sozialwissenschaftlicher und biologischer Forschungsansätze (z.B. „Sociophysiology“ [5]). Um der Komplexität seelischer Krankheiten gerecht zu werden, könnten derartige Ansätze helfen, deren Entstehung und Verlauf besser zu verstehen. Dies stellt die Eigenständigkeit sozialpsychiatrischer Forschung nicht infrage, denn deren Expertise ist genauso wie das Fachwissen biologischer Forschung bei solchen Fragestellungen nötig. Manche hier bewusst nicht genannten Publikationen zeigen, wie vereinfachend gedacht wird, wenn biologische Forscher sozialwissenschaftliche Aspekte in ihre Studien einbeziehen - und umgekehrt, wenn Sozialwissenschaftler biologische Fragestellungen inkludieren. Nichtsdestotrotz gibt es positive Beispiele solcher Kooperationen [7].

Die Ursachen und Entstehungsmechanismen psychischer Erkrankungen besser zu verstehen, ist eine wesentliche Grundlage sowohl für präventive Ansätze als auch für verbesserte Behandlungsmethoden. Solch kooperative Projekte würden also längerfristig den Kranken zugutekommen. Abgesehen von den erwähnten Kooperationen braucht es aber jene Bereiche, wo die sozialpsychiatrische Forschung genuine Beiträge leisten kann und muss. Im Folgenden seien nur einige beispielhaft erwähnt: 
- Auch wenn wir heute prinzipiell ein breites Spektrum an wirksamen Interventionen im außerstationären Bereich zur Verfügung haben, wird dieses Spektrum oft nicht entsprechend genützt und es gibt zahlreiche Hinweise auf Mängel in der Versorgung [8-11]. Bezüglich der Ursachen weshalb es bislang nicht ausreichend gelang, das aus Studien Bekannte flächendeckend umzusetzen, gibt es zahlreiche Spekulationen, allerdings gibt es nur wenige aktuelle Studien, die sich der Frage nach den Hindernissen für die Umsetzung widmen [12]. Auch der Frage, ob hier geschlechtsspezifische Unterschiede zu berücksichtigen sind, wird kaum nachgegangen [13-15].

- Die stationäre psychiatrische Versorgung hat sich in den letzten 3 Jahrzehnten dramatisch verändert [16]. Die Versorgungsforschung hatte zu Recht in den letzten Jahrzehnten einen starken Focus auf die außerstationäre Versorgung gelegt. Im Vergleich dazu aber wird recht selten untersucht, ob die Versorgung für jene, die eine stationäre Behandlung benötigen, auch im Sinne der Kranken funktioniert [17,18].

- Noch immer wissen wir nicht, ob psychische Erkrankungen wirklich zunehmen, wie oft postuliert wird [19,20]. Da die bisherigen Studiendesigns die Analyse dieser Frage häufig nur mit großen Einschränkungen erlauben, sind wir hier oft auf Spekulationen angewiesen.

- Es ist eine allgemein bekannte Tatsache, dass die Zahl älterer Menschen in den nächsten Jahrzehnten deutlich steigen wird. Mit Ausnahme von Demenzen und Depressionen wissen wir aber kaum etwas über die epidemiologische Verteilung und die Versorgung der Kranken [21,22]. Außerdem ist nicht auszuschließen, dass bei älteren Menschen andere ätiologische und pathogenetische Mechanismen als bei jüngeren zum Tragen kommen.

- Noch immer gelingt es uns nicht, häufige psychische Erkrankungen frühzeitig zu erkennen und zu behandeln $[23,24]$. Neben den traditionellen Überlegungen gibt es kaum neue Ansätze, wie z. B. gestuftes Screening oder innovative statistische Methoden [25]. Gerade hier wird es nötig sein, über den „Tellerrand“ konventioneller Forschungsdesigns hinaus innovative Wege zu gehen, um dieses Defizit zu beseitigen.

- Die Zahl jener, die aus einem anderen Kulturkreis stammen, ist seit Jahren im Steigen begriffen. Auch wenn die Frage nach transkulturellen Aspekten in der Behandlung und Versorgung in letzter Zeit vermehrt Aufmerksamkeit fand [26-29], ist unser Wissen hier sehr lückenhaft. Gerade eine Disziplin wie die Sozialpsychiatrie, die den sozialen Kontext zu ihren zentralen Fragestellungen zählt, sollte sich verstärkt den Fragen des Einflusses von kulturellen Phänomenen auf die Entstehung und den Verlauf psychischer Erkrankungen widmen.

Diese wenigen Themenkomplexe zeigen nur beispielhaft, dass die sozialpsychiatrische Forschung ihre eigenen Aufgaben und Fragestellungen hat. Wenn wir die Behandlung und die Lebenssituation der Kranken verbessern wollen, wird es aber unumgänglich sein, mit der biologischen und der psychotherapeutischen Psychiatrie zusammenzuarbeiten - in der Wissenschaft wie im klinischen Alltag. Es braucht in der sozialpsychiatrischen Forschung also beides, die Kooperation mit den anderen Bereichen der Psychiatrie und die Fokussierung auf die eigenen Themenbereiche.

\section{Literatur}

1 Richter D, Hoffmann $H$. Nach der pharmakologischen Euphorie - Eine neue Chance für die Entwicklung und Umsetzung psychosozialer Interventionen. Psychiat Prax 2009; 36: 357-358

2 Pharoah F, Mari J, Rathbone J et al. Family intervention for schizophrenia. Cochrane Database of Systematic Reviews 2006; Issue 4. Art. No. CD000088

3 Hyman SE. The millennium of mind, brain and behavior. Arch Gen Psychiatry 2000; $57: 88-89$

4 Eisenberg $L$. The social construction of the human brain. Am J Psychiatry 1995; 152: 1563-1575

5 Gardner R. Psychiatry needs a basic science titled sociophysiology (editorial). Biol Psychiatry 1996; 38: 833-834

6 Kendler KS. The nature of genetic influence on behavics. Am J Psychiat 2006; 136: 1683-1694

7 Tsuang MT, Bar JL, Stone WS et al. Gene-environment interactions in mental disorders. World Psychiatry 2004; 3: 73-83

8 Kaiser G, Krautgartner $M$, Alexandrowicz $R$ et al. Die Übereinstimmungsvalidität des „Carers' Needs Assessment for Dementia“ (CNAD). Neuropsychiatr 2005; 19: 134-140

9 Krautgartner $M$, Unger A, Friedrich $F$ et al. Risiken für Depressivität bei den Angehörigen Schizophrenie-Kranker. Neuropsychiatr 2005; 19: $148-154$

10 Aichhorn W, Santeler S, Stelzig-Schöler R et al. Prävalenz psychiatrischer Erkrankungen bei wohnungslosen Jugendlichen. Neuropsychiatr 2008; 22: 180-188

11 Jäger $M$, Rössler $W$. Informeller Zwang zur Verbesserung der Behandlungsbereitschaft psychiatrischer Patienten. Neuropsychiatr 2009; 23: $206-215$

12 Sibitz I, Schrank B, Amering A. Nutzung der Angehörigenrunde. Neuropsychiatr 2009; 23: 26-34

13 Wancata J, Freidl M, Friedrich F et al. Gibt es geschlechtsspezifische Unterschiede in der elterlichen Betreuung von Schizophrenie-Kranken? Neuropsychiatr 2008; 22: 83-91

14 Schmidt R, Assem-Hilger E, Benke T et al. Geschlechtsspezifische Unterschiede der Alzheimer Demenz. Neuropsychiatr 2008; 22: 1-15

15 Hausmann A, Rutz W, Meise U. Frauen suchen Hilfe - Männer sterben! Ist die Depression wirklich weiblich? Neuropsychiatr 2008; 22: 43-48

16 Rittmannsberger H, Sartorius N, Brad M et al. Changing aspects of psychiatric inpatient treatment: a census investigation in five European countries. European Psychiatry 2004; 19: 483-488

17 Hausner H, Wittmann M, Haen E et al. Psychopharmakoepidemiologie: Qualitative Unterschiede zwischen stationären und ambulanten Verordnungsstrategien. Psychiat Prax 2008; 35: 337-342

18 Rittmannsberger $H$, Lindner $H$, Zaunmüller $T$. Herkunft psychiatrischer Patienten im UbG-Bereich einer städtischen Region. Neuropsychiatr 2008; 22: 28-34

19 Richter D, Berger K, Reker T. Nehmen psychische Störungen zu? Eine systematische Literaturübersicht. Psychiat Prax 2008; 35: 321-330

20 Mauz E, Jacobi F. Psychische Störungen und soziale Ungleichheit im Geburtskohortenvergleich. Psychiat Prax 2008; 35: 343-352

21 Riedel-Heller SG, Busse A, Angermeyer MC. The state of mental health in old-age across the old European Union: a systematic review. Acta Psychiatr Scand 2006; 113: 388-401

22 Castro-Costa E, Dewey $M$, Stewart $R$ et al. Prevalence of depressive symptoms and syndromes in ten European countries - the SHARE study. Brit J Psychiatry 2007; 191: 393-401

23 Donath C, Gräßel E, Großfeld-Schmitz M et al. Diagnostik und Therapie von Demenzerkrankungen in der hausärztlichen Praxis: ein StadtLand-Vergleich. Psychiat Prax 2008; 35: 142-145

24 Sielk M, Altiner A, Janssen B et al. Prävalenz und Diagnostik depressiver Störungen in der Allgemeinarztpraxis. Ein kritischer Vergleich zwischen PHQ-D und hausärztlicher Einschätzung. Psychiat Prax 2009; 36: $169-174$

25 Alexandrowicz $R$, Weiss M, Marquart B et al. Zur Validität eines zweistufigen Screenings am Beispiel des Depressionsscreening. Psychiat Prax 2008; 35: 294-301

26 Grube M. Sind Zwangseinweisungen in psychiatrische Kliniken bei Migranten häufiger? Psychiat Prax 2009; 36: 67-71

27 Glaesmer H, Wittig U, Brähler E et al. Sind Migranten häufiger von psychischen Störungen betroffen? Eine Untersuchung an einer repräsentativen Stichprobe der deutschen Allgemeinbevölkerung? Psychiat Prax 2009; 36: 16-22

28 Yilmaz TA, Riecher-Rössler A. Suizidversuche in der ersten und zweiten Generation der ImmigrantInnen aus der Türkei. Neuropsychiatr 2008; 22: 261-267

29 Ritter K, Stompe T. Die Akzeptanz von Suizidmotiven - ein Schlüssel zu den Unterschieden nationaler Suizidraten? Neuropsychiatr 2008; 22: 172-179 